\title{
Beliefs for Human-Unique Social Learning
}

\author{
Hilary Richardson \\ The University of Edinburgh \\ hilary.richardson@ed.ac.uk
}

Target article: Jonathan Phillips, Wesley Buckwalter, Fiery Cushman, Ori Friedman, Alia Martin, John Turri, Laurie Santos, and Joshua Knobe. (2020). Knowledge before belief. Behavioral and Brain Sciences, 1-37. 


\begin{abstract}
Phillips et al. argue that understanding what others know is central to social cognition across species and that this understanding underlies human-unique accumulation and transmission of cultural knowledge. Knowledge representations can't be both what we have in common with our evolutionary ancestors and what sets us apart from them. Belief representations are necessary for human-unique social learning.
\end{abstract}


In the course of investigating whether infants and non-human primates represent beliefs, developmental and comparative psychologists generated compelling evidence that these populations represent knowledge. However, evidence that knowledge representations are important for social life across species does not diminish the importance of belief representations for humans. Humans and non-human primates - who share the capacity to represent knowledge nonetheless have dramatically different capacities for accumulating cultural knowledge. While the capacity to represent knowledge is important for cultural transmission, it is insufficient for humanunique social learning. We must also reason about beliefs.

Phillips et al. argue that knowledge representations - rather than belief representations - support human-unique accumulation and transmission of cultural knowledge because they are clearly in place during infancy and early childhood. In contrast, empirical evidence suggests that social behaviors inherent to human-unique accumulation and transmission of culture develop relatively slowly during early childhood. For example, humans have unique capacities for teaching and learning, aligning perspectives through persuasion, and creating and motivating action towards shared goals. These three social behaviors are honed during early childhood and are supported by mental representations that are tagged with their epistemic history and are not necessarily factive - i.e., beliefs.

Reasoning about informants' beliefs enables us to engage in selective social learning and pedagogy: we consider and evaluate the epistemic history of our and others' beliefs to decide who to learn from and what to teach (e.g., Heyes, 2018). As children get older, they increasingly prefer to learn from more accurate informants (Harris et al., 2012; Harris \& Corriveau, 2011) and the preference to learn from more accurate - but not physically stronger - informants is predicted by their capacity to reason about diverse beliefs, controlling for age (Brosseau-Liard et al., 2015). As children reason more flexibly about beliefs, they come to understand that teaching is guided by a teacher's belief about the knowledge gap - rather than the actual knowledge gap - between themselves and their learners (Ziv \& Frye, 2004). Theory of mind development in early childhood is linked to children becoming better teachers themselves: children who pass explicit false belief tasks selectively present evidence that not only provides knowledge to their learner, but also corrects their learner's particular false belief (controlling for age and numerical conservation reasoning; Bass et al., 2019). Humans do not simply teach and learn to fill gaps in knowledge; we predict, consider, and correct false beliefs.

In addition to correcting others' beliefs, we strategically manipulate them (e.g., Weinstein, 1969). Young children increasingly use the beliefs of the persuadee (e.g., Tricia thinks puppies bite) to tailor their persuasive arguments (e.g., tell Tricia that puppies are gentle rather than quiet; Bartsch et al., 2007). The ability to generate persuasive arguments improves during early childhood and correlates with theory of mind reasoning, controlling for age and language ability (Peterson et al., 2018; Slaughter et al., 2013). Children with disproportionate deficits in theory of mind reasoning show reduced performance on persuasion tasks (Peterson et al., 2018). Skillfully persuading others to adopt our own mental representations requires reasoning about the content and epistemic history of theirs.

Phillips et al. convincingly argue that belief representations are better suited for action prediction than knowledge representations. Accordingly, belief representations also underlie humans' ability 
to organize and motivate others' actions toward shared goals. False belief reasoning correlates with production of joint proposals and assignment of roles during pretend play, controlling for age and language abilities (Astington \& Jenkins, 1995) and six-year-old children use first- and secondorder belief representations to coordinate on tasks with their peers (Grueneisen et al., 2015).

Regardless of exactly when children or infants begin to represent others' beliefs (e.g., PoulinDubois et al., 2018), there is ample evidence that belief representations are used more flexibly and in service of increasingly sophisticated social behaviors - including social behaviors inherent to human-unique accumulation and transmission of knowledge - during early childhood. This continued development reflects genuine conceptual change in theory of mind representations, rather than a gradual unmasking of competence as language and executive functions improve. Childhood theory of mind reasoning is predicted by earlier theory of mind capacities over and above these other skills (Peterson \& Wellman, 2019; Wellman et al., 2011; Richardson et al., unpublished data) and is mirrored by continued development in brain regions that support social cognition (Richardson et al., 2018), including specialization of the right temporoparietal junction for reasoning about mental states (beliefs, desires, emotions; Richardson et al., 2020). The capacity to reason about beliefs - deliberately, with slow and gradual improvement during childhood, and with consequences for populations for whom this is challenging - is intrinsic to human-unique accumulation and transmission of cultural knowledge.

Acknowledging a relatively slower developmental trajectory for human-unique accumulation and transmission of cultural knowledge additionally allows formal education to play a role. In many societies, school provides a venue for children to learn not only how to read and write but also how to become a citizen of their community and culture (Zigler \& Trickett, 1978). Explicit false belief reasoning in early childhood predicts school readiness (controlling for age, language, IQ, attention shifting, and executive functions, Blair \& Razza, 2007; for review, see Astington \& Pelletier, 2005), suggesting that, in addition to enabling increasingly sophisticated social behaviors, ongoing theory of mind development enables children to capitalize on institutions specifically in place for the accumulation and transmission of cultural knowledge.

As Phillips et al. propose, one important goal for future theory of mind research is to offer a description of early developing, evolutionarily shared, automatic, and preserved capacities - like knowledge representations - and the social behaviors that they can and cannot support. A second and equally important goal is to offer a description of ongoing conceptual change in childhood which includes the development of theory of mind capacities that are core to human-unique intelligence and culture. 


\section{Acknowledgments}

I am grateful to Ashley Thomas, Shari Liu, and Lindsey Powell for feedback, and to Rebecca Saxe for encouragement. 


\section{References}

Astington, J. W., \& Jenkins, J. M. (1995). Theory of mind development and social understanding. Cognition \& Emotion, 9(2-3), 151-165.

Astington, J. W., \& Pelletier, J. (2005). Theory of mind, language, and learning in the early years: Developmental origins of school readiness. The Development of Social Cognition and Communication, 205-230.

Bartsch, K., London, K., \& Campbell, M. D. (2007). Children's attention to beliefs in interactive persuasion tasks. Developmental Psychology, 43(1), 111.

Bass, I., Gopnik, A., Hanson, M., Ramarajan, D., Shafto, P., Wellman, H., \& Bonawitz, E. (2019). Children's developing theory of mind and pedagogical evidence selection. Developmental Psychology, 55(2), 286.

Blair, C., \& Razza, R. P. (2007). Relating effortful control, executive function, and false belief understanding to emerging math and literacy ability in kindergarten. Child Development, $78(2), 647-663$.

Brosseau-Liard, P., Penney, D., \& Poulin-Dubois, D. (2015). Theory of mind selectively predicts preschoolers' knowledge-based selective word learning. British Journal of Developmental Psychology, 33(4), 464-475.

Grueneisen, S., Wyman, E., \& Tomasello, M. (2015). “I know you don’t know I know...” Children use second-order false-belief reasoning for peer coordination. Child Development, 86(1), 287-293.

Harris, P. L., \& Corriveau, K. H. (2011). Young children's selective trust in informants. Philosophical Transactions of the Royal Society B: Biological Sciences, 366(1567), $1179-1187$. 
Harris, P. L., Corriveau, K. H., Pasquini, E. S., Koenig, M., Fusaro, M., \& Clément, F. (2012). Credulity and the development of selective trust in early childhood. Foundations of Metacognition, 193-210.

Heyes, C. (2018). Cognitive Gadgets: The Cultural Evolution of Thinking. Harvard University Press.

Peterson, C. C., Slaughter, V., \& Wellman, H. M. (2018). Nimble negotiators: How theory of mind (ToM) interconnects with persuasion skills in children with and without ToM delay. Developmental Psychology, 54(3), 494.

Peterson, C. C., \& Wellman, H. M. (2019). Longitudinal theory of mind (ToM) development from preschool to adolescence with and without ToM delay. Child Development, 90(6), $1917-1934$.

Poulin-Dubois, D., Rakoczy, H., Burnside, K., Crivello, C., Dörrenberg, S., Edwards, K., Krist, H., Kulke, L., Liszkowski, U., \& Low, J. (2018). Do infants understand false beliefs? We don't know yet-A commentary on Baillargeon, Buttelmann and Southgate's commentary. Cognitive Development, 48, 302-315.

Richardson, H., Koster-Hale, J., Caselli, N., Magid, R., Benedict, R., Olson, H., Pyers, J., \& Saxe, R. (2020). Reduced neural selectivity for mental states in deaf children with delayed exposure to sign language. Nature Communications, 11(1), 1-13.

Richardson, H., Lisandrelli, G., Riobueno-Naylor, A., \& Saxe, R. (2018). Development of the social brain from age three to twelve years. Nature Communications, 9(1), 1027.

Slaughter, V., Peterson, C. C., \& Moore, C. (2013). I can talk you into it: Theory of mind and persuasion behavior in young children. Developmental Psychology, 49(2), 227. 
Weinstein, E. A. (1969). The development of interpersonal competence. Handbook of Socialization Theory and Research, 753-775.

Wellman, H. M., Fang, F., \& Peterson, C. C. (2011). Sequential progressions in a theory-of-mind scale: Longitudinal perspectives. Child Development, 82(3), 780-792.

Zigler, E., \& Trickett, P. K. (1978). IQ, social competence, and evaluation of early childhood intervention programs. American Psychologist, 33(9), 789.

Ziv, M., \& Frye, D. (2004). Children's understanding of teaching: The role of knowledge and belief. Cognitive Development, 19(4), 457-477.

Ziv, M., Solomon, A., Strauss, S., \& Frye, D. (2016). Relations between the development of teaching and theory of mind in early childhood. Journal of Cognition and Development, $17(2), 264-284$. 